\title{
Epicardial and transverse sinus fat pad near left atrium appendage; Role of 3D echocardiography
}

\author{
reza badalabadi ${ }^{1}$, mohammad sahebjam ${ }^{1}$, mehdi badalabadi ${ }^{1}$, and kaveh hosseini ${ }^{1}$ \\ ${ }^{1}$ Affiliation not available
}

September 11, 2020

\begin{abstract}
Pericardial fat pad in transverse sinus adjacent to LAA is a rare condition that may resemble LAA thrombosis especially in suspected cases. More TEE angulations and 3D TEE can confirm the diagnosis.
\end{abstract}

\section{Key clinical message}

Pericardial fat pad in transverse sinus adjacent to LAA is a rare condition that may resemble LAA thrombosis especially in suspected cases. More TEE angulations and 3D TEE can confirm the diagnosis.

Key words: Epicardial fat pad, three-dimension echocardiography

\section{Introduction}

The anatomy of pericardial cavity consists of two sinuses; transverse and oblique sinuses. A recess in pericardial cavity adjacent to pulmonary vein and also another one in transverse sinus near pulmonary artery have been detected in 70-80\% of general population. Transverse sinus (TS) is located anterior to the left atrium (LA) and almost posterior to the aortic root. In some people the tip of the LA appendage (LAA) may be located posterior, inferior or even into the pericardial sinus. Many anatomic variations in LAA have been depicted in the literature. Sometimes puncture of the inter-atrial septum may enter the transverse pericardial sinus and endanger the aortic root $[1,2]$.

Pericardial fat pad in transverse sinus adjacent to LAA is a rare condition that may resemble LAA thrombosis especially in suspected cases. Atrial fibrillation and severe mitral stenosis (MS) change the morphology and geometry of the LA/LAA. LAA itself has many complex variations.

Herein we present a rare anatomic variation in a patient with severe MS who was candidate for percutaneous transvenous mitral commissurotomy (PTMC) called transverse sinus fat pad (TSFP). In trans-esophageal Echocardiographic (TEE) views even an expert operator may be mistaken at fist if he or she is not familiar with TSFP.

\section{Case presentation}

A 60 years old woman with history of dyspnea on exertion from 2 years ago presented to the Tehran Heart Center[3]. After initial evaluations in trans-thoracic echocardiography (TTE) severe mitral stenosis with score Wilkins[4] score 7 was diagnosed. She was planned to undergo TEE for further evaluation and probable PTMC in this admission. She was on Inderal 20 daily from 6 months ago and furosemide 20 daily was added recently due to her dyspnea. TEE was done without complication after local anesthesia with $1.5 \mathrm{mg}$ midazolam. Severe MS was seen and low LAA velocity $(28.2 \mathrm{~cm} / \mathrm{s})$ was noted, Figure 1; Panel A. Suspicious mass was seen near LAA and was first consumed to be a LAA thrombosis. After more angulations of the probe and other off-axis views it was considered as a well- defined mass-like lesion in TS, Figure 1; 
Panel B; measuring $2.35 \mathrm{~cm} * 2.65 \mathrm{~cm}$, Figure 1; Panel C. In this figure, one can also notice thick epicardial fat pad. The Echogenisity of lesion within TS is exactly like the epicardial fat around right ventricle (RV), Figure 1, Panel C thin arrow.

Three-dimension mapping was performed with live 3D wide angled full volume TEE. Echogenicity and features of the lesion was against thrombosis and was in favor of fat tissue, Figure 1; Panel D. Hence, TSFP was diagnoses. Videos of $3 \mathrm{D}$ and 2D TEE imaging has been attached as a supplementary file.

\section{Discussion}

The transverse sinus is a pericardial reflection which is between the arterial mesocardium and the venous mesocardium. Existence of epicardial fat between ascending aorta and transverse sinus is a normal finding however, presence of fat pad in the sinus adjacent to LAA is rare and misleading. Epicardial fat surrounding the left pulmonary artery recess but not in TS was previously reported by Johner et al in Cardiology journal 2019[2]. They were not familiar with this anatomic variation around LAA and cancelled the cardioversion for atrial fibrillation (AF) due to LAA thrombosis!

As far as we know this is the third case of TSFP reported in literature. First case was introduced by Chhabra et al [5] and the second case was presented with Pergolini et al both in 2019[6]. None of these studies evaluated the patient with 3D technique. In our experience 3D mapping is a useful modality since the echodensity features in 3D mapping of thrombosis is different from fat pad and an expert echocardiologist can differentiate these two easily. In present patient the increased epicardial fat pad around RV was also noted. The similarity of the density between RV fat pad and TS lesion is another clue that this is not a thrombosis.

Although 2D TEE has higher resolution than 3D mapping due to higher frame rate, 3D mapping technology allows more comprehensive evaluation of LAA anatomy and its nearby structures. Its ability to differentiate the borders between LAA and adjacent structures made 3D TEE a feasible and reliable tool before invasive procedures such as LAA device closure.

Low velocity in LAA increases the risk of thrombosis formation however the absence of smoky pattern is in contrast with thrombosis in LAA. Several TEE angulations can prove the TSFP originates from outside the LAA in contrast with thrombosis. In addition, utilization of other imaging modalities such as CT scan and MRI may help diagnosis this anatomic variation.

\section{Conclusion}

Sometimes epicardial fat pad fills the transverse sinus (TSFP) near LAA and resembles thrombosis. This mistake may preclude or delay further procedures such as cardioversion or PTMC. Hence, along-side 2D imaging, 3D TEE can precisely delineate the complex structure of LA and LAA.

Funding: None

Informed consent was taken from patient.

CONFLICT OF INTEREST

The authors have no conflict of interest to declare.

Ethical Approval Code: IR-TUMS.VCR.REC.1399.123

AUTHOR CONTRIBUTION

RMB and KH: Gathered all clinical materials and images. MS: performed 3D TEE and scientific supervision. MMB: Performed TEE. MS: revised the manuscript. KH: Reviewed the literature and drafted the initial version of manuscript.

\section{References}


1. Ho, S.Y., J.A. Cabrera, and D. Sanchez-Quintana, Left atrial anatomy revisited. Circulation: Arrhythmia and Electrophysiology, 2012.5 (1): p. 220-228.

2. Johner, N., et al., Epicardial fat mimicking left atrial appendage thrombus. Cardiology journal, 2019. 26 (4): p. 418-419.

3. Poorhosseini, H. and S.H. Abbasi, The Tehran Heart Center . 2018, Oxford University Press.

4. Martinez-Rios, M.A., et al., Percutaneous mitral commissurotomy. Cardiology in review, 1999. 7 (2): p. 108-116.

5. Chhabra, L., et al., Transverse sinus fat pad may masquerade as left atrial appendage thrombus. Journal of electrocardiology, 2019.56 : p. 43-45.

6. Pergolini, A., et al., The transverse pericardial sinus: an unpleasant third wheel. Kardiologia Polska, 2019. 78 (2): p. 165-166.

Figure Legend

Figure 1. 2D and 3D TEE imaging, A: TEE short axis of aorta at $80^{\circ}$ angulation reveals reduced LAA velocity. B: TEE short axis aorta at $64^{\circ}$ angulation shows fat pad in transverse.C: well-define echo-density $2.35 \mathrm{~cm} * 2.65 \mathrm{~cm}$ between aorta and LAA, epicardial fat pad around right ventricle is also evident (thin arrow). D: 3D TEE imaging reveals fat like density around LAA

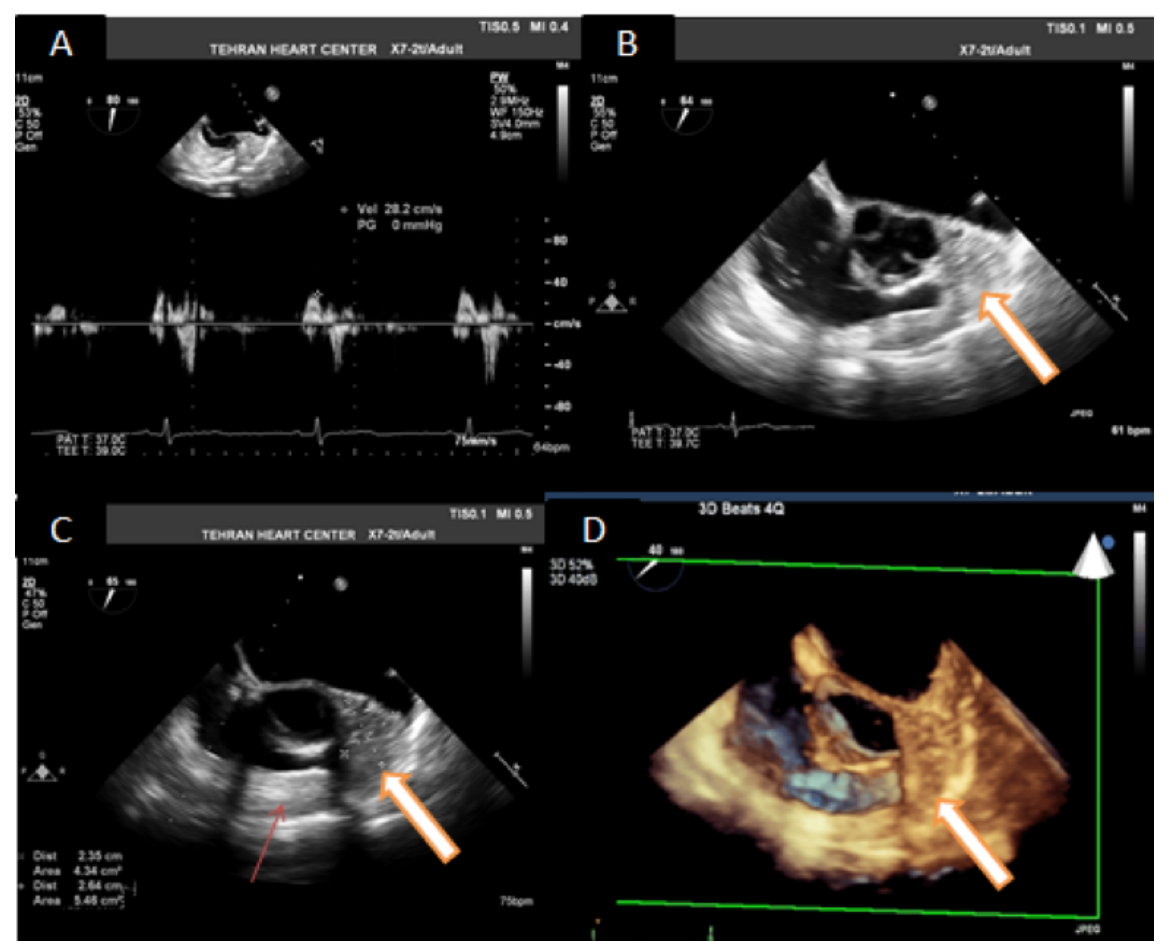

